\title{
The role of cancer metabolism in defining the success of oncolytic viro-immunotherapy
}

\author{
Arthur Dyer ${ }^{1}$, Sally Frost ${ }^{1}$, Kerry Fisher ${ }^{1}$ and Len Seymour ${ }^{1,2}$ \\ 1 Department of Oncology, University of Oxford, Oxford, UK \\ 2 len.seymour@oncology.ox.ac.uk, Old Road Campus Research Building, Department of \\ Oncology, University of Oxford, OX37DQ
}

\section{Declaration of Interests}

LS and KF holds equity in PsiOxus Therapeutics Ltd. All the other authors declare that they have no conflict of interest.

\section{Funding Information}

AD, KF and LS are funded by Cancer Research UK (CRUK, C557/A17720). S.F. is supported by MRC Centre for Doctoral Training grant number MR/N013468/1. A.D. is supported by a Graduate Scholarship from Jesus College, Oxford.

\section{Author Contributions}

Arthur Dyer: Conceptualisation, writing - original draft, writing - reviewing \& editing Sally Frost: Conceptualisation, writing - original draft, writing - reviewing \& editing Kerry Fisher: Conceptualisation, supervision Len Seymour: Conceptualisation, writing - reviewing \& editing, Supervision, Project administration.

\begin{abstract}
Oncolytic viruses infect, replicate in, and kill cancer cells selectively without harming normal cells. The rapidly expanding clinical development of oncolytic virotherapy is an exciting interdisciplinary field that provides insights into virology, oncology, and immunotherapy. Recent years have seen greater focus on rational design of cancer-selective viruses together with strategies to exploit their immunostimulatory capabilities, ultimately to develop powerful oncolytic cancer vaccines. However, despite great interest in the field, many important experiments are still conducted under optimum conditions in vitro, with many nutrients present in excess and with cellular stress kept to a minimum. Whilst this provides a convenient platform for cell culture, it bears little relation to the typical conditions found within a tumour in vivo, where cells are often subject to a range of metabolic and environmental stresses. Viral infection and cancer will both lead to production of metabolites that are also not present in media in vitro. Understanding how oncolytic viruses interact with cells exposed to more representative metabolic conditions in vitro represents an under-explored area of study that could provide valuable insight into the intelligent design of superior oncolytic viruses and help bridge the gap between bench and
\end{abstract}


bedside. This review summarises the major metabolic pathways altered in cancer cells, during viral infection and highlights possible targets for future studies.

\section{Introduction}

\section{Modified metabolism as a hallmark of cancer}

It has long been documented that cancer cells have altered cellular programming which results in increased survival, growth and proliferation leading to tumour formation and progression. These key changes were documented in the seminal review "The Hallmarks of Cancer" by Hanahan and Weinberg in 2000 which highlighted the underlying principles that govern the transformation of normal cells to cancer cells [1]. This review highlighted the genetic and epigenetic changes that induce stem-cell-like properties, such as unlimited cell division and blocked differentiation. In 2011 the same authors released an updated list with two new hallmarks; immune evasion and deregulated metabolism, both of which are fundamental to the success of oncolytic virotherapy [2].

One well studied feature of metabolic reprogramming in cancer is the "Warburg Effect" in which tumour cells increase glucose consumption and lactate secretion, even in the presence of oxygen [3]. This provides several key advantages to cancer cells. Notably the rate of metabolism through aerobic glycolysis occurs 10-100 times faster than oxidative phosphorylation such that the amount of ATP synthesised over a given period is comparable or even greater [4]. Against this background, the metabolic plasticity in most cancer cells allows for alternative metabolites (such as glutamine) to be used for biosynthetic purposes and it has been shown that malignant cells require a high metabolic flux to synthesise building blocks such as nucleotides, proteins and lipids. This anabolic phenotype induces a unique microenvironment that includes decreased concentrations of nutrients and oxygen, and increased levels of harmful metabolic waste products such as the production of lactate which inhibits many immune cell functions. All of which contributes towards formation of an "immunologically cold" cancer, which is difficult to treat and is associated with poor prognosis.

\section{Lytic viruses as anticancer agents}

Oncolytic virotherapy has recently received renewed interest since promising clinical results for the first agent (Imlygic) which received its product licence in the USA and Europe in 2015 $[5,6]$. The current upsurge of interest in oncolytic viruses (OVs) reflects the culmination of incremental scientific progress gained over several years that is now being translated into important clinical progress [6-9].

Many wild-type viruses show an intrinsic selectivity for replication within cancer cells. The mechanism underlying this is a topic of intense discussion and is likely to reflect the activated nature of tumour cells, providing a cellular phenotype that is conducive to efficient virus replication. Many publications highlight that there appears to be considerable overlap between the hallmarks of cancer and the hallmarks of virus infection. 
As viruses are obligate intracellular pathogens, they need to hijack cellular processes to create a favourable environment for efficient production of infectious progeny. In many ways their demands for macromolecular synthesis appear similar to those of a proliferating or cancer cell. Metabolomic analysis of virally infected cells was first conducted with cells infected with human cytomegalovirus (HCMV) [10]. During the lytic portion of its lifecycle, HCMV was found to induce a range of metabolic changes including an upregulation of the tricarboxylic acid (TCA) cycle, nucleotide biosynthesis, and glycolysis. Such alterations are now known to be critical for replication of many viruses, including Kaposi's sarcomaassociated herpesvirus, herpes simplex virus 1 , adenovirus, hepatitis $C$ virus, human immunodeficiency virus and dengue virus [10-15].

As with tumour cells, infection of cells with viruses induces a unique metabolic microenvironment including decreased concentrations of nutrients, such as glucose, glutamine, arginine, tryptophan and decreased oxygen, and an increase in metabolic waste products, such as lactate, glutamate, and reactive oxygen species (ROS).

\section{Oncolytic virotherapy and metabolism}

The success of oncolytic virotherapy relies on the ability of the virus to enter and replicate successfully within tumour cells that are exposed to the many stressful environments listed above. However, there are surprisingly few publications investigating how oncolytic viruses cope with unfavourable metabolic conditions. As a notable example, the levels of glucose used in vitro typically range from 5-25 mM whereas in clinical cancers the glucose concentrations can be as low as 0.1-0.4 mM, even with an average blood concentration of around $6 \mathrm{mM}$ [16]. Equally, lactate levels intratumourally can rise to levels of $40 \mathrm{mM}$, which is far higher than normally present in in vitro conditions [17].

In nearly all published studies, cancer cells were grown in these non-pathological conditions with nutrients like glucose far in excess of what is to be found even in the blood circulation. The impact of high levels of glucose on these experiments is a key consideration for future studies because it is crucial to test new drugs targeting cancer cells under physiologically relevant conditions. One such example is that of metformin (a drug currently being tested for a wide range of cancer treatments) which has been shown to be more effective in enhancing the sensitivity of cancer cells to chemotherapy only when cells are grown in reduced glucose conditions [18]. In 2016, Pusapati et al showed that weaning cancer cell lines off of glucose makes them significantly more susceptible to co-treatment with mTORc inhibitors and anti-glycolysis drugs [19].

\section{Glucose and glutamine metabolism}

The recent advances in the field of metabolomics have led to a much deeper understanding of metabolism of non-cancerous cells, tumour metabolism and how pathogenic agents hijack cellular metabolism in order to meet their own energetic and biosynthetic demands. Both cancerous and virus-infected cells show a phenotype of increased uptake and catabolism of nutrients. Infectious viruses of many types have been shown to reprogram 
various aspects of host cell central carbon metabolism including increased glycolysis, elevated pentose phosphate pathway and increased lipid and amino acid synthesis. Whilst several viruses upregulate consumption of key nutrients like glucose and/or glutamine and converge on a similar metabolic pathway, the precise metabolic changes induced by specific viruses is often context-dependent and can vary even within families of viruses or cell types that are infected. Whilst we do not aim to encompass the entire field of viral metabolism in this brief review, we have focused on the metabolic challenges associated with some of the viruses that present promising opportunities for oncolytic virotherapy.

\section{DNA Viruses}

\section{Herpesviruses}

In the 1950s Eagle and Habel showed that replication of poliovirus (a positive single stranded RNA virus) in HeLa cells was abrogated in culture media composed solely of a balanced salt solution. Upon addition of glucose to the salt solution viral proliferation and viral loads were restored to normal levels, suggesting that glycolysis was essential to allow poliovirus replication [20]. These findings were subsequently confirmed in primary monkey kidney cells where an increase in lactate in the media during the first two hours of infection was shown [21,22]. As early as 1962 it was shown that replication of Herpes Simplex Virus 1 (HSV-1, an enveloped double-stranded DNA virus) was also dependent on glucose in HeLa cells [23].

Members of the Herpesviridae family of viruses are, to date, the most studied viruses in terms of metabolism. For HSV-1, in 1962 it was shown that in the absence of glucose, viral entry into host cells was not affected but that no infectious progeny was produced [23]. It was shown that HSV-1 virion production did not decrease in the presence of 2-Deoxy-dglucose (2DG, a competitive inhibitor of glucose metabolism) but that production of infectious particles was severely impaired, possibly due to glycosylation changes in the viral glycoproteins on the envelope of the virus [24]. Similar results were also shown using another alphaherpesvirus, pseudorabies [25].

When the host cell response to infection with HSV1 was analysed via metabolomics it was found that with HSV-1, only around $1 \%$ of glycolytic TCA cycle intermediates were derived from glycolysis and that TCA intermediates were instead provided largely by a flow of glutamine via $\alpha$-ketoglutarate [12]. A possible explanation for this dramatic change in metabolism is that glucose uptake may have been increased to feed the pentose phosphate pathway to support increased nucleotide synthesis, leaving a demand for other metabolites to be produced from an alternative source such as glutamine $[27,28]$.

These results highlight a few important lessons. Firstly, the metabolic demands of one virus in a particular cell type is not the requirement for all viruses, even if they are closely related 
and secondly, despite these differences, the metabolic requirements of these viruses can be defined to quite specific accuracy.

\section{Vaccinia Virus}

Vaccinia virus, a cytoplasm-based enveloped double stranded DNA poxvirus, provides an interesting case point in the current literature regarding metabolomics during viral infection. Studies on vaccinia virus infected human foreskin fibroblasts showed no increase of glycolytic intermediates, and this led Fontaine et al to hypothesise that glycolysis is not necessary for vaccinia virus replication [29]. This was confirmed by the removal of glucose from the media of vaccinia virus infected cells, resulting in no effect on the levels of replication and production of infectious progeny. This indicated that utilisation of glycolysis for viral replication is evidently not universal [29]. Further studies have shown that vaccinia virus replication is highly dependent on glutamine and that restriction of glutamine or inhibition of glutaminolysis inhibits the vaccinia virus lifecycle [29,30]. It has recently been shown that this dependence on glutamine was due to expression of the viral protein C16 which stabilises hypoxia inducible factor 1 alpha (HIF1 $\alpha$ ) [31].

\section{Adenoviruses}

Adenoviruses are a diverse family of non-enveloped double stranded DNA viruses. Shortly after they were discovered, it was observed that infection with adenoviruses causes an increase in glycolysis and lactic acid production [32-34]. Many members of the adenovirus family have been shown to induce glycolysis through expression of an early viral gene, E4ORF1, which interacts with Myc causing it to translocate to the nucleus and specifically upregulate metabolic genes involved in glutamine and glucose metabolism [34,35].

Interestingly, in cells that have a low glycolytic rate, knockdown of c-Myc decreases viral titres but not in cells with a high glycolytic rate [35]. The observation that adenoviruses can respond to glycolytic alterations differently in cells that have a high glycolytic rate compared to those with a low glycolytic rate was confirmed in a 2018 paper released from our laboratory which showed that less glycolytic cells tend to be less permissive to viral oncolysis and that restriction of glycolysis through pharmaceutical inhibition or through repeated culture in low glucose conditions lead to an improved viral lifecycle [34].

With respect to adenoviruses and glutamine metabolism, it is evident from the research listed above that glutamine metabolism is vital for viral replication and that inhibition of glutamine metabolism dramatically inhibits viral replication, a fate that adenoviruses also share with HSV-1 and influenza [36]. However boosting glutamine metabolism and, more specifically reductive carboxylation (a metabolic pathway whereby glutamine can be metabolised outside of mitochondria without the requirement for oxygen) can improve viral oncolysis [34,36].

Interestingly, human adenovirus species D type 36 and 31 are prevalent in obese individuals and infection with type 36 human adenovirus has been associated with metabolic changes in animal models [37-39]. These metabolic changes have been linked again to the early viral 
protein E4ORF1 and are the subject of many research papers investigating whether there is a causal link between the expression of this protein and obesity with some claiming that adenovirus 36 infection promotes weight gain both in vitro and in vivo [37-41]. further research conducted in murine and avian models showed that infection with Ad36 resulted in a statistically significant weight gain [38] however, infection with Ad36 also resulted in an improved glycaemic control $[37,42,43]$. These alterations were later found to be attributable to the E4ORF1 gene and its interactions with phosphatidyl inositol 3-kinase (PI3K) and with MYC [44-46] leading many research groups to suggest the potential of E4ORF1 as a template for developing antidiabetic therapies because of its ability to strongly control glycolysis to favour the pentose phosphate pathway. The metabolic reprogramming of host cells by adenoviruses is reviewed in greater detail in a 2019 paper by Prusinkiewicz and Mymryk highlighting the considerable overlaps between the metabolic profiles of adenovirus infections and the Warburg effect [47].

\section{RNA Viruses}

\section{HIV}

One virus which highlights some of the difficulties in studying viral metabolomics is the Human Immunodeficiency Virus 1 (HIV-1). HIV-1 is an enveloped single stranded positive sense RNA virus that is classified as a non-transforming lentivirus. A metabolomic study of HIV-1 infected macrophages and CD4 ${ }^{+} \mathrm{T}$ Cells showed an interesting result. In macrophages, which generally maintain a long-term (latent) infection, infection with HIV-1 resulted in a decreased uptake of glucose and a decrease in glycolytic metabolite accumulation [14]. In $\mathrm{CD}^{+}{ }^{+} \mathrm{T}$ Cells, which generally support an acute lytic infection, the presence of HIV-1 resulted in the opposite metabolic profile with infected cells showing increased glycolysis $[14,48]$. These findings highlight the importance of the host cell model used to study metabolomics and echo findings in other models suggesting that latency and acute infection can show markedly different metabolic profiles in closely related viruses $[49,50]$.

\section{Influenza}

Many other viruses either directly induce glycolysis during infection or cause host cells to upregulate glycolysis at various stages during infection such as several strains of influenza $A$, a single stranded, segmented negative strand RNA virus, which induces glycolysis at a relatively late stage of infection (coinciding with the onset of apoptosis) [51]. In 1959, the glycolysis inhibitor 2-DG was shown to inhibit viral replication in vitro in line with the findings that influenza increases glycolysis [52]. This finding, however, was contrasted in 2016 by Wang et al who showed that treatment with 2-DG in an in vivo influenza model decreased overall survival by increasing viral replication [53]. The authors suggested that this decrease in host survival was likely due to the fact that 2-DG treatment will result in deregulated unfolded protein response (UPR). These contrasting differences between a cellular level of control by the virus and an organism level of homeostasis and metabolism highlight the divergent metabolic needs of the different cell types within an organism. 


\section{Rhinovirus}

Rhinovirus, a small RNA virus which belongs to the picornaviridae family and is the causative agent of the common cold. It has been shown that, as with most viruses, a response of host cells to infection with rhinoviruses is an increased glucose uptake and that the virus is dependent on both glutamine and glucose for optimal replication [54]. It is interesting to note that, unlike most DNA viruses mentioned above, this increase in glucose uptake occurs as early as 1.5 hours post infection meaning that this response to infection occurs before viral proteins can be translated meaning this is a host cell response to viral infection and not caused by expression of a viral protein.

\section{Rhabdoviruses}

Rhabdoviridae is a family of enveloped group V viruses (single stranded negative RNA genomes) of which we will focus on Vesicular Stomatitis Virus (VSV) because this virus is the most common member of the family used in oncolytic virotherapy. VSV is able to replicate in a wide number of cancer cells both in normoxic and hypoxic conditions making it an attractive platform for oncolytic virotherapy [55]. Previous studies have shown that VSV replication is enhanced in cancer cells when they are grown in malignant ascites fluid as these conditions increased the glycolysis of certain cell lines meaning these cells increased lactate production and elevated the use of glutamine as the predominant carbon source [56]. Indeed, inhibition of glycolysis or glutaminolysis has been shown to decrease oncolytic VSV replication however this can be rescued by addition of oxidative TCA intermediates when glycolysis is inhibited or through addition of $\alpha$-ketoglutarate when glutaminolysis is inhibited [56].

\section{Paramyxoviruses}

Viruses in the family of Paramyxoviridae are negative-sense, single stranded RNA viruses that include measles, mumps and Newcastle disease virus (NDV). Little is currently known about how NDV alters metabolism in cells, however, in 2014, Deng et al suggested that NDV might reduce the activity of host glycolysis by decreasing the glycolytic enzyme phosphoglycerate kinase (PGK) [57]. Despite the paucity of information on the metabolic alterations of cells infected with NDV, the virus seems highly selective for cancer cells and is displaying promise as an oncolytic agent [58]. As with NDV, relatively little is known about how measles viruses affect the metabolism of their host cells but a paper in 2014 reported that infection of cells with the oncolytic measles virus Edmonston strain (MV-Edm) upregulates glycolysis under aerobic conditions (the Warburg effect) in glioblastoma cells, which was evidenced by increased glucose uptake, lactate production, and LDHA expression [59]. 


\section{Viral metabolic demands conclusion}

Overall, although the metabolic response of host cells to viral infections have overlapping commonalities, the metabolic demands for each virus are varied and even closely related viruses can have different requirements. However, each virus has clear metabolic demands and metabolic consequences for the microenvironment, summarised in table 1.

Despite this seemingly widespread increase in glucose uptake during viral infection there are some marked differences between both the causes of this increase and the benefits to the viruses. Some viruses actively increase glucose uptake whereas in other cases, the host cells increase glucose uptake in response to the presence of the virus. It is also worth noting that despite infection with viruses causing an upregulation of glucose uptake, inhibition of glycolysis or removal or restriction of glucose during infection can have a wide variety of effects on the virus lifecycle, as will be discussed later.

Glutamine metabolism in general and the reliance of virally infected cells upon glutamine is less studied than glucose in the field of virology and even less so in oncolytic virotherapy. In general, many cancer cells are considered to be "glutamine addicted" meaning they use glutamine as a source of metabolites, in part compensating for the lack of oxidative glucose metabolism. Glutamine is often catabolised by reductive carboxylation and can be used by cells for a range of purposes including producing ATP, amino acids, nucleotides, fatty acids, mTOR activation, the hexosamine biosynthesis pathway, as well as protecting cells against ROS [60-62]. Because of these factors it is undoubtedly an essential nutrient for many OV and further research needs to be done to understand whether altering glutamine metabolism can improve the outcome of oncolytic virotherapy.

\section{Restriction of metabolites during cancer therapy}

The reduction of nutrients or the removal of metabolites from media, or the use of metabolic inhibitors provides important insights for the field of virology in general and holds particular importance for the field of oncolytic virotherapy. In humans, a blood glucose concentration of $6 \mathrm{mM}$ is considered average and a blood glucose level of about $7.1 \mathrm{mM}$ is considered hyperglycaemic. In many cases, the glucose concentrations observed in bodily compartments can be much lower than the levels observed in the blood.

There is an increasing amount of scientific literature in both pre-clinical and clinical settings showing that fasting can sensitise malignant cells to cancer treatments whilst at the same time protecting non-malignant cells in a phenomenon that is referred to as the differential stress response (DSR) [63]. The principal reasoning that is postulated to explain the DSR is that during tumourigenesis various mutations are gained including the ability to proliferate in unfavourable conditions without the presence of external growth factor signalling and that these cells therefore become prone to stressful conditions such as chemotherapy when deprived of nutrients as they are not able to respond to the protective signals generated by fasting [2]. This response is in contrast to non-malignant cells which, during calorie restrictions or fasting have been shown to respond favourably (figure 2). Preclinical studies 
have shown that intermittent fasting can extend lifespans, delay type II diabetes and cardiovascular and neurodegenerative diseases as well as reducing cancer rates [64-68].

There are various clinical trials and pre-clinical projects investigating the effects of calorie restriction (reducing intake to $75 \%$ fewer calories) and short-term starvation (no food intake) on cancer treatments due to the apparent weakness of cancer cells to restricting nutrients and the benefits to normal tissues. Preliminary reports have found that fasting for between two to five days can protect patients from the side effects of chemotherapy without causing chronic weight loss [68]. This is in contrast to calorie restriction which requires weeks or months to be effective and causes more modest changes in glucose levels in both humans and rodents [69].

With respect to preclinical investigations into the effects of the DSR in cancer treatments, Raffaghello et al published in 2008 that starvation in mice allowed researchers to increase the tolerable dose of chemotherapy up to three times higher than the maximal dose approved in humans [70]. Lee et al published in 2012 that 15 out of 17 mammalian cancer cell lines tested are sensitised to various chemotherapeutics by fasting 24 hours prior to and 24 hours post-chemotherapy [71]. This paper also showed that two cycles of fasting in mice was as effective as two cycles of chemotherapy alone in reducing tumour burden and that the combination of starvation plus chemotherapy was the most effective regimen [71].

To date there have been a small number of clinical trials that have researched the effect of combined fasting and chemotherapy in patients mainly focussing on the reduction in side effects of chemotherapy. Safdie et al in 2009 reported a case study with 10 patients showing reduced chemotherapeutic side effects when fasting 48-140 h prior and 5-56 h post treatment [71]. Other similar reports are reviewed by De Groot et al [69]. There are many larger clinical trials currently ongoing to determine the possible benefits of fasting with respect to treatment efficacy, adverse events, quality of life and changes in metabolic, hormone and inflammatory response (NCT00936364, NCT01802346, NCT02710721, NCT03162289, NCT03340935, NCT03595540, NCT03709147, NCT03700437, NCT01175837).

\section{Restriction of metabolites during oncolytic virus therapy}

There have been only a limited number of studies investigating the effects of restricting metabolites or fasting on oncolytic virotherapy, although they have all shown highly promising results. The first such paper written by Esaki et al showed that transient fasting improved the replication of oncolytic herpes virus in glioblastoma models with striking results shown both in vitro and in vivo [72]. In 2019, Scheubeck et al reported that low glucose, low serum starvation has the potential to enhance measles virus-mediated oncolysis efficacy in HT-29 cells, in terms of a synergistic effect [73].

We reported in 2019 that restriction of glucose or inhibition of glycolysis improved viral DNA replication, viral protein synthesis and the rate of cell lysis after infection with an oncolytic adenovirus in glucose-dependent cell lines and that removal of glucose does not inhibit infection in highly permissive cancer cells [34]. We also reported that cells that were previously poorly permissive to oncolytic adenovirus became highly permissive after repeated culture in low glucose media or in the presence of glycolytic inhibitors. 
Despite little being known about how paramyxoviruses alter metabolism, there have been some highly promising results studying the benefits of restricting metabolism during infection with OVs in the family Paramyxoviridae. In 2014, it was reported that that attenuated oncolytic measles virus Edmonston strain (MV-Edm) caused glioblastoma cells to shift to high-rate aerobic glycolysis; this adaptation was blocked by dichloroacetate (DCA), an inhibitor of glycolysis, leading to profound cell death of cancer cells but not of normal cells. DCA enhanced viral replication by mitigating mitochondrial antiviral signalling proteinmediated innate immune responses [59]. Two groups have investigated the inhibition of glycolysis during oncolytic treatment with NDV. They independently found that 2-DG and NDV synergise to kill breast cancer cells by inhibition of the glycolysis pathway through GAPDH downregulation [74], and that the pyruvate dehydrogenase kinase inhibitor DCA not only promotes NDV replication in a similar manner but, excitingly appeared to improve the immune response to NDV oncolysis by removing immunosuppressive microenvironmental factors such as kynurenine due to IDO1 expression and lactate [75].

These papers highlight the possibilities of combining OVs with metabolism-altering treatments and are only possible through rigorous understanding of the metabolic demands of each virus platform. What is true for one virus will not be true for all and so any use of glycolytic inhibitors or intermittent fasting needs to be evaluated for each viral platform. Further uses of understanding metabolic interactions and OVs will be discussed below.

\section{Future Directions}

As discussed above, reduction of metabolites to more clinically relevant levels can have a marked effect upon how cancer cells respond to stressors such as OVs. Whilst modelling precisely the environment encountered in vivo can seem daunting or even impossible, there are methods that can be adopted to, at least attempt to bridge the gap between current culture conditions and those encountered in a tumour. The reduction of metabolites such as glucose and/or glutamine to much lower levels is a simple prospect that that be readily incorporated into most in vitro, cancer models. Many groups have found promising results through repeated culture of cells in low metabolite conditions because, at first, some cells will not respond well to a decline in these previously abundant metabolites and likely require time to adjust their metabolism to be able to cope with the more stressful conditions $[34,76]$. Nevertheless, a number of studies have shown that previously highly glycolytic cell lines when cultured for even a small amount of time in low glucose conditions completely alter their metabolic profiles [77-79].

Gaining an understanding of the metabolic requirements of cells infected with your OV of choice is of key importance for a number of reasons. Firstly, it could allow for a more targeted therapy of cancers. Many techniques such as positron emission tomography (PET) imaging and single-cell analysis have advanced such that we can gain insights into the metabolic landscape of tumours before treatment and this along with advancements in genotyping could allow for screening of patients with a tumour that will support OV treatment. Likewise, 
understanding the metabolic requirements of cells infected with any given OV can highlight limiting metabolites that are required for effective oncolysis allowing a supplementation of these through dietary means, through co-treatment with metabolic drugs designed to increase specific metabolic pathways, or through the design of armed OVs that express enzymes or drugs designed to target the metabolic environment.

In considering cancer as a whole, it would be remiss to not consider the effects of altered metabolism following oncolytic virus infection on the immune status of tumours. Little work is currently conducted into the effects of OVs upon the immune system specifically focussed on the effects of altered metabolism and how that changes the microenvironment. A large number of OVs will increase both nutrient competition and metabolic waste accumulation. Whilst it is true that viral infection has been shown to increase the presence of immunostimulatory markers such as DAMPs and PAMPs and can cause immunogenic cell death of host cells, it remains unclear if this is able to outcompete the immunosuppressive environment that viruses may encourage due to exaggeration of the Warburg effect or an increase in suppressive metabolites already found in the tumour microenvironment. Understanding what level of effect these factors exert over immune cell function will only improve the design of OVs especially those armed to encourage immune cell stimulation.

Some promising examples of harnessing metabolism to improve oncolytic virotherapy have been published in the past two years. One such example is the finding that inhibiting glycolysis during infection with oncolytic Newcastle disease virus appears to improve the immune response by removing immunosuppressive factors such as IDO1 and lactate [75]. An exciting advancement in the field of immunometabolism was published recently highlighting the possibility of using OVs as a vector to alter metabolism in favour of viral replication and improved immune cell stimulatory phenotypes. Rivadeneira et al in 2019 reported that the immune response to OVs was incomplete due to metabolic insufficiencies induced by the tumour microenvironment and this was exacerbated by the presence of the virus. The authors then showed that the adipokine, leptin, was able to metabolically reprogramme T cells and that engineering OVs to produce leptin within the tumour induced complete responses in tumour-bearing mice and supported memory development in the tumour infiltrate [80].

\section{Conclusions}

Cancer cells, immune cells and the surrounding milieu that make up the tumour microenvironment all play a critical role in the success or failure of any anti-cancer drug and this is particularly true of oncolytic viruses which rely on the behaviour of cancer cells for their success. Recently there have been a number of papers showing that cancer cell lines behave differently depending upon the metabolic environment in which they are cultured $[18,19]$. Several studies have also indicated that either through starvation or through 
restriction of certain nutrients, cancer cells can become more vulnerable to lysis with OVs or can improve the ability of the virus to replicate within these cells with dramatic effects being seen in situations or cell lines where OVs previously struggled to perform. Certainly, even a relatively simple step of reducing glucose concentrations in vitro can dramatically alter the response of cancer cells to stressors and, we believe, should be considered a vital step in bridging the gap between the bench and bedside.

Despite the fact that metabolic requirements differ based on which virus is used, each OV requires a specific, yet definable, intracellular metabolic environment and they provide an obvious and, as yet, underexplored platform with which to produce these environments through the expression of metabolic reprogramming agents. Furthermore, the immune response to OV infection may be dampened through immune cell competition for nutrients with virally infected cells or through the increase in metabolic waste from virally infected cells. OVs which can alter the tumour microenvironment to favour both virus infection and to create a more immune-stimulating environment represents an exciting step forward in the field of viro-immunotherapy with some promising results beginning to emerge to support this theory $[34,59,72-75]$. 


\section{Differential Stress Response}
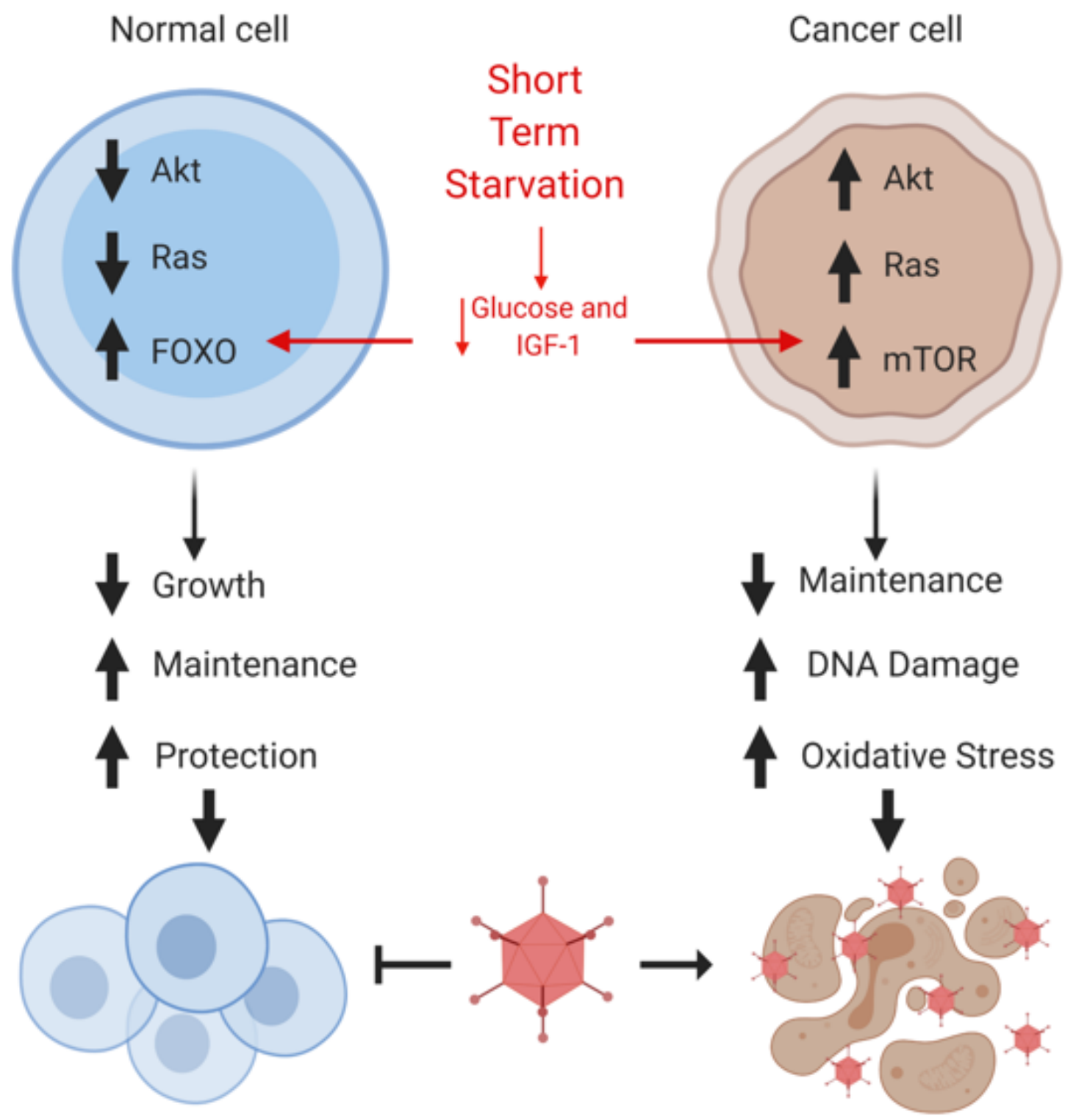

Differential Stress Response in cancer treatment. Simplified schematic representation of the differer stress response. Short term starvation decreases serum levels of glucose and insulin-like growth fact (IGF-1) which affect downstream growth regulators including mammalian target of rapamycin (mTOF Akt, Ras and Forkhead Box O proteins (FOXO). In cancer cells, these regulators are activated in respor the short-term starvation increasing oxidative stress and decreasing cell maintenance leading to DNA damage and making them more susceptible to lysis by oncolytic viruses whereas the opposite occurs non-cancerous cells. 


\section{References}

[1] D. Hanahan, R.A. Weinberg, The hallmarks of cancer., Cell. 100 (2000) 57-70. https://doi.org/10.1007/s00262-010-0968-0.

[2] D. Hanahan, R.A. Weinberg, Hanahan D, Weinberg RA. Hallmarks of cancer: The next generation. Vol. 144, Cell. 2011. p. 646-74. Ilmarks of cancer: The next generation, Cell. 144 (2011) 646-674.

[3] O. Warburg, On Respiratory Impairment in Cancer Cells, Science (80-. ). 124 (1956) 269-70. https://doi.org/10.1126/science.124.3215.267.

[4] A.A. Shestov, X. Liu, Z. Ser, A.A. Cluntun, Y.P. Hung, L. Huang, D. Kim, A. Le, G. Yellen, J.G. Albeck, J.W. Locasale, Quantitative determinants of aerobic glycolysis identify flux through the enzyme GAPDH as a limiting step, Elife. 3 (2014) 1-18.

https://doi.org/10.7554/eLife.03342.

[5] FDA Approves First Oncolytic Virus Therapy, Oncol. Times. 37 (2015) 36. https://doi.org/10.1097/01.COT.0000475724.97729.9e.

[6] A. Dyer, R. Baugh, S.L. Chia, S. Frost, Iris, E.J. Jacobus, H. Khalique, T.D. Pokrovska, E.M. Scott, W.K. Taverner, L.W. Seymour, J. Lei, Turning cold tumours hot: oncolytic virotherapy gets up close and personal with other therapeutics at the 11th Oncolytic Virus Conference, Cancer Gene Ther. (2018) 1. https://doi.org/10.1038/s41417-018-0042-1.

[7] S.J. Russell, K.-W. Peng, J.C. Bell, Oncolytic virotherapy, Nat. Biotechnol. 30 (2012) 658-670. https://doi.org/10.1038/nbt.2287.

[8] E.A. Chiocca, S.D. Rabkin, Oncolytic Viruses and Their Application to Cancer Immunotherapy, Cancer Immunol. Res. 2 (2014) 295-300. https://doi.org/10.1158/2326-6066.CIR-14-0015.

[9] L.W. Seymour, K.D. Fisher, Oncolytic viruses: finally delivering., Br. J. Cancer. 114 (2016) 35761. https://doi.org/10.1038/bjc.2015.481.

[10] J. Munger, S.U. Bajad, H.A. Coller, T. Shenk, J.D. Rabinowitz, Dynamics of the Cellular Metabolome during Human Cytomegalovirus Infection, PLoS Pathog. 2 (2006) e132. https://doi.org/10.1371/journal.ppat.0020132.

[11] T. Delgado, E.L. Sanchez, R. Camarda, M. Lagunoff, Global Metabolic Profiling of Infection by an Oncogenic Virus: KSHV Induces and Requires Lipogenesis for Survival of Latent Infection, PLoS Pathog. 8 (2012) e1002866. https://doi.org/10.1371/journal.ppat.1002866.

[12] L. Vastag, E. Koyuncu, S.L. Grady, T.E. Shenk, J.D. Rabinowitz, Divergent Effects of Human Cytomegalovirus and Herpes Simplex Virus-1 on Cellular Metabolism, PLoS Pathog. 7 (2011) e1002124. https://doi.org/10.1371/journal.ppat.1002124.

[13] D.L. Diamond, A.J. Syder, J.M. Jacobs, C.M. Sorensen, K.-A. Walters, S.C. Proll, J.E. McDermott, M.A. Gritsenko, Q. Zhang, R. Zhao, T.O. Metz, D.G. Camp, K.M. Waters, R.D. Smith, C.M. Rice, M.G. Katze, Temporal Proteome and Lipidome Profiles Reveal Hepatitis C Virus-Associated Reprogramming of Hepatocellular Metabolism and Bioenergetics, PLoS Pathog. 6 (2010) e1000719. https://doi.org/10.1371/journal.ppat.1000719.

[14] J.A. Hollenbaugh, J. Munger, B. Kim, Metabolite profiles of human immunodeficiency virus infected CD4+ T cells and macrophages using LC-MS/MS analysis, Virology. 415 (2011) 153159. https://doi.org/10.1016/J.VIROL.2011.04.007.

[15] G. Birungi, S.M. Chen, B.P. Loy, M.L. Ng, S.F.Y. Li, Metabolomics Approach for Investigation of 
Effects of Dengue Virus Infection Using the EA.hy926 Cell Line, J. Proteome Res. 9 (2010) 6523-6534. https://doi.org/10.1021/pr100727m.

[16] A. Hirayama, K. Kami, M. Sugimoto, M. Sugawara, N. Toki, H. Onozuka, T. Kinoshita, N. Saito, A. Ochiai, M. Tomita, H. Esumi, T. Soga, Quantitative Metabolome Profiling of Colon and Stomach Cancer Microenvironment by Capillary Electrophoresis Time-of-Flight Mass Spectrometry, Cancer Res. 69 (2009) 4918-4925. https://doi.org/10.1158/0008-5472.CAN08-4806.

[17] A. Brand, K. Singer, G.E. Koehl, M. Kolitzus, G. Schoenhammer, A. Thiel, C. Matos, C. Bruss, S. Klobuch, K. Peter, M. Kastenberger, C. Bogdan, U. Schleicher, A. Mackensen, E. Ullrich, S. Fichtner-Feigl, R. Kesselring, M. Mack, U. Ritter, M. Schmid, C. Blank, K. Dettmer, P.J. Oefner, P. Hoffmann, S. Walenta, E.K. Geissler, J. Pouyssegur, A. Villunger, A. Steven, B. Seliger, S. Schreml, S. Haferkamp, E. Kohl, S. Karrer, M. Berneburg, W. Herr, W. Mueller-Klieser, K. Renner, M. Kreutz, LDHA-Associated Lactic Acid Production Blunts Tumor Immunosurveillance by T and NK Cells, Cell Metab. 24 (2016) 657-671. https://doi.org/10.1016/j.cmet.2016.08.011.

[18] H. Yu, X. Bian, D. Gu, X. He, Metformin Synergistically Enhances Cisplatin-Induced Cytotoxicity in Esophageal Squamous Cancer Cells under Glucose-Deprivation Conditions, (2016). https://doi.org/10.1155/2016/8678634.

[19] R. V. Pusapati, A. Daemen, C. Wilson, W. Sandoval, M. Gao, B. Haley, A.R. Baudy, G. Hatzivassiliou, M. Evangelista, J. Settleman, mTORC1-Dependent Metabolic Reprogramming Underlies Escape from Glycolysis Addiction in Cancer Cells, Cancer Cell. 29 (2016) 548-562. https://doi.org/10.1016/J.CCELL.2016.02.018.

[20] H. EAGLE, K. HABEL, The nutritional requirements for the propagation of poliomyelitis virus by the HeLa cell., J. Exp. Med. 104 (1956) 271-87.

http://www.ncbi.nlm.nih.gov/pubmed/13345971 (accessed January 8, 2019).

[21] S. BARON, H.B. LEVY, Some metabolic effects of poliomyelitis virus on tissue culture., Nature. 178 (1956) 1230-1. http://www.ncbi.nlm.nih.gov/pubmed/13387671 (accessed January 8, 2019).

[22] H.B. Levy, S. Baron, The Effect of Animal Viruses on Host Cell Metabolism II. Effect of Poliomyelitis Virus on GLycolysis and Uptake of Glycine by Monkey Kidney Tissue Cultures, J. Infect. Dis. 100 (1957) 109-118. https://doi.org/10.1093/infdis/100.2.109.

[23] V.J. LEWIS, L. V SCOTT, Nutritional requirements for the production of herpes simplex virus. I. Influence of glucose and glutamine of herpes simplex virus production by HeLa cells., J. Bacteriol. 83 (1962) 475-82. http://www.ncbi.nlm.nih.gov/pubmed/14464909 (accessed February 8, 2018).

[24] R.J. Courtney, S.M. Steiner, M. Benyesh-Melnick, Effects of 2-deoxy-d-glucose on herpes simplex virus replication, Virology. 52 (1973) 447-455. https://doi.org/10.1016/00426822(73)90340-1.

[25] H. Ludwig, R. Rott, Effect of 2-deoxy-D-glucose on herpesvirus-induced inhibition of cellular DNA synthesis., J. Virol. 16 (1975) 217-21. http://www.ncbi.nlm.nih.gov/pubmed/168399 (accessed January 8, 2019).

[26] J.L. Abrantes, C.M. Alves, J. Costa, F.C.L. Almeida, M. Sola-Penna, C.F.L. Fontes, T.M.L. Souza, Herpes simplex type 1 activates glycolysis through engagement of the enzyme 6phosphofructo-1-kinase (PFK-1), Biochim. Biophys. Acta - Mol. Basis Dis. 1822 (2012) 11981206. https://doi.org/10.1016/j.bbadis.2012.04.011. 
[27] M.P. Landini, Early Enhanced Glucose Uptake in Human Cytomegalovirus-infected Cells, J. Gen. Virol. 65 (1984) 1229-1232. https://doi.org/10.1099/0022-1317-65-7-1229.

[28] Y. Saito, R.W. Price, Enhanced regional uptake of 2-deoxy-D-[14C]glucose in focal herpes simplex type 1 encephalitis: autoradiographic study in the rat., Neurology. 34 (1984) 276-84. http://www.ncbi.nlm.nih.gov/pubmed/6538267 (accessed January 8, 2019).

[29] K.A. Fontaine, R. Camarda, M. Lagunoff, Vaccinia Virus Requires Glutamine but Not Glucose for Efficient Replication, J. Virol. 88 (2014) 4366-4374. https://doi.org/10.1128/JVI.03134-13.

[30] M.D. Greseth, P. Traktman, De novo Fatty Acid Biosynthesis Contributes Significantly to Establishment of a Bioenergetically Favorable Environment for Vaccinia Virus Infection, PLoS Pathog. 10 (2014) e1004021. https://doi.org/10.1371/journal.ppat.1004021.

[31] M. Mazzon, C. Castro, L.D. Roberts, J.L. Griffin, G.L. Smith, A role for vaccinia virus protein C16 in reprogramming cellular energy metabolism, J. Gen. Virol. 96 (2015) 395-407. https://doi.org/10.1099/vir.0.069591-0.

[32] T.N. Fisher, H.S. Ginsberg, Accumulation of Organic Acids by HeLa Cells Infected with Type 4 Adenovirus., Exp. Biol. Med. 95 (1957) 47-51. https://doi.org/10.3181/00379727-95-23113.

[33] K.R. Rozee, L.J. Ottey, C.E. van Rooyen, SOME METABOLIC EFFECTS OF ADENOVIRUS INFECTION IN HELA CELLS, Can. J. Microbiol. 3 (1957) 1015-1020. https://doi.org/10.1139/m57-113.

[34] A. Dyer, B. Schoeps, S. Frost, P.G. Jakeman, E.M. Scott, J. Freedman, L.W. Seymour, Antagonism of glycolysis and reductive carboxylation of glutamine potentiates activity of oncolytic adenoviruses in cancer cells, Cancer Res. (2018). https://doi.org/10.1158/00085472.CAN-18-1326.

[35] M. Thai, N.A. Graham, D. Braas, M. Nehil, E. Komisopoulou, S.K. Kurdistani, F. McCormick, T.G. Graeber, H.R. Christofk, Adenovirus E4ORF1-induced MYC activation promotes host cell anabolic glucose metabolism and virus replication, Cell Metab. 19 (2014) 694-701. https://doi.org/10.1016/j.cmet.2014.03.009.

[36] M. Thai, S.K. Thaker, J. Feng, Y. Du, H. Hu, T. Ting Wu, T.G. Graeber, D. Braas, H.R. Christofk, MYC-induced reprogramming of glutamine catabolism supports optimal virus replication, Nat. Commun. 6 (2015) 8873. https://doi.org/10.1038/ncomms9873.

[37] N. V Dhurandhar, B.A. Israel, J.M. Kolesar, G.F. Mayhew, M.E. Cook, R.L. Atkinson, Increased adiposity in animals due to a human virus., Int. J. Obes. Relat. Metab. Disord. 24 (2000) 98996. http://www.ncbi.nlm.nih.gov/pubmed/10951537 (accessed August 1, 2019).

[38] N. V Dhurandhar, L.D. Whigham, D.H. Abbott, N.J. Schultz-Darken, B.A. Israel, S.M. Bradley, J.W. Kemnitz, D.B. Allison, R.L. Atkinson, Human adenovirus Ad-36 promotes weight gain in male rhesus and marmoset monkeys., J. Nutr. 132 (2002) 3155-60. https://doi.org/10.1093/jn/131.10.3155.

[39] R.L. Atkinson, N. V Dhurandhar, D.B. Allison, R.L. Bowen, B.A. Israel, J.B. Albu, A.S. Augustus, Human adenovirus-36 is associated with increased body weight and paradoxical reduction of serum lipids, Int. J. Obes. 29 (2005) 281-286. https://doi.org/10.1038/sj.ijo.0802830.

[40] I. Bil-Lula, A. Krzywonos-Zawadzka, G. Sawicki, M. Woźniak, An infection of human adenovirus 31 affects the differentiation of preadipocytes into fat cells, its metabolic profile and fat accumulation, J. Med. Virol. 88 (2016) 400-407. https://doi.org/10.1002/jmv.24362.

[41] H.-N. Na, O. Dubuisson, V. Hegde, J.-H. Nam, N. V Dhurandhar, Human adenovirus Ad36 and 
its E4orf1 gene enhance cellular glucose uptake even in the presence of inflammatory cytokines., Biochimie. 124 (2016) 3-10. https://doi.org/10.1016/j.biochi.2014.11.005.

[42] R. Krishnapuram, E.J. Dhurandhar, O. Dubuisson, H. Kirk-Ballard, S. Bajpeyi, N. Butte, M.S. Sothern, E. Larsen-Meyer, S. Chalew, B. Bennett, A.K. Gupta, F.L. Greenway, W. Johnson, M. Brashear, G. Reinhart, T. Rankinen, C. Bouchard, W.T. Cefalu, J. Ye, R. Javier, A. Zuberi, N. V. Dhurandhar, Template to improve glycemic control without reducing adiposity or dietary fat, Am. J. Physiol. - Endocrinol. Metab. 300 (2011). https://doi.org/10.1152/ajpendo.00703.2010.

[43] W.Y. Lin, O. Dubuisson, R. Rubicz, N. Liu, D.B. Allison, J.E. Curran, A.G. Comuzzie, J. Blangero, C.T. Leach, H. Göring, N. V. Dhurandhar, Long-term changes in adiposity and glycemic control are associated with past adenovirus infection, Diabetes Care. 36 (2013) 701-707. https://doi.org/10.2337/dc12-1089.

[44] E.J. Dhurandhar, O. Dubuisson, N. Mashtalir, R. Krishnapuram, V. Hegde, N. V. Dhurandhar, E4orf1: A novel ligand that improves glucose disposal in cell culture, PLoS One. 6 (2011). https://doi.org/10.1371/journal.pone.0023394.

[45] E.J. Dhurandhar, R. Krishnapuram, V. Hegde, O. Dubuisson, R. Tao, X.C. Dong, J. Ye, N. V. Dhurandhar, E4orf1 Improves Lipid and Glucose Metabolism in Hepatocytes: A Template to Improve Steatosis \& Hyperglycemia, PLoS One. 7 (2012). https://doi.org/10.1371/journal.pone.0047813.

[46] R. Krishnapuram, E.J. Dhurandhar, O. Dubuisson, V. Hegde, N. V. Dhurandhar, DoxycyclineRegulated 3T3-L1 Preadipocyte Cell Line with Inducible, Stable Expression of Adenoviral E4orf1 Gene: A Cell Model to Study Insulin-Independent Glucose Disposal, PLoS One. 8 (2013). https://doi.org/10.1371/journal.pone.0060651.

[47] M.A. Prusinkiewicz, J.S. Mymryk, Metabolic Reprogramming of the Host Cell by Human Adenovirus Infection, Viruses. 11 (2019) 141. https://doi.org/10.3390/v11020141.

[48] E.Y. Chan, J.N. Sutton, J.M. Jacobs, A. Bondarenko, R.D. Smith, M.G. Katze, Dynamic host energetics and cytoskeletal proteomes in human immunodeficiency virus type 1-infected human primary CD4 cells: analysis by multiplexed label-free mass spectrometry., J. Virol. 83 (2009) 9283-95. https://doi.org/10.1128/JVI.00814-09.

[49] K. Wang, Y. Hoshino, K. Dowdell, M. Bosch-Marce, T.G. Myers, M. Sarmiento, L. Pesnicak, P.R. Krause, J.I. Cohen, Glutamine supplementation suppresses herpes simplex virus reactivation, J. Clin. Invest. 127 (2017) 2626-2630. https://doi.org/10.1172/JCl88990.

[50] E.L. Sanchez, P.A. Carroll, A.B. Thalhofer, M. Lagunoff, Latent KSHV Infected Endothelial Cells Are Glutamine Addicted and Require Glutaminolysis for Survival, PLOS Pathog. 11 (2015) e1005052. https://doi.org/10.1371/journal.ppat.1005052.

[51] J.B. Ritter, A.S. Wahl, S. Freund, Y. Genzel, U. Reichl, Metabolic effects of influenza virus infection in cultured animal cells: Intra- and extracellular metabolite profiling, BMC Syst. Biol. 4 (2010) 61. https://doi.org/10.1186/1752-0509-4-61.

[52] E.D. Kilbourne, Inhibition of influenza virus multiplication with a glucose antimetabolite (2deoxy-D-glucose), Nature. 183 (1959) 271-272. https://doi.org/10.1038/183271b0.

[53] A. Wang, S.C. Huen, H.H. Luan, S. Yu, C. Zhang, J.D. Gallezot, C.J. Booth, R. Medzhitov, Opposing Effects of Fasting Metabolism on Tissue Tolerance in Bacterial and Viral Inflammation, Cell. 166 (2016) 1512-1525.e12. https://doi.org/10.1016/j.cell.2016.07.026.

[54] G.A. Gualdoni, K.A. Mayer, A.M. Kapsch, K. Kreuzberg, A. Puck, P. Kienzl, F. Oberndorfer, K. 
Frühwirth, S. Winkler, D. Blaas, G.J. Zlabinger, J. Stöckl, Rhinovirus induces an anabolic reprogramming in host cell metabolism essential for viral replication, Proc. Natl. Acad. Sci. U. S. A. 115 (2018) E7158-E7165. https://doi.org/10.1073/pnas.1800525115.

[55] S.A. Felt, V.Z. Grdzelishvili, Ecent advances in vesicular stomatitis virus-based oncolytic virotherapy: A 5-year update, J. Gen. Virol. 98 (2017) 2895-2911.

https://doi.org/10.1099/jgv.0.000980.

[56] Y. Zhou, F. Wen, P. Zhang, R. Tang, Q. Li, Vesicular stomatitis virus is a potent agent for the treatment of malignant ascites, Oncol. Rep. 35 (2016) 1573-1581.

https://doi.org/10.3892/or.2015.4522.

[57] X. Deng, Y. Cong, R. Yin, G. Yang, C. Ding, S. Yu, X. Liu, C. Wang, Z. Ding, Proteomic analysis of chicken peripheral blood mononuclear cells after infection by Newcastle disease virus, J. Vet. Sci. 15 (2014) 511-517. https://doi.org/10.4142/jvs.2014.15.4.511.

[58] J. Kalyanasundram, A. Hamid, K. Yusoff, S.L. Chia, Newcastle disease virus strain AF2240 as an oncolytic virus: A review, Acta Trop. 183 (2018) 126-133.

https://doi.org/10.1016/j.actatropica.2018.04.007.

[59] C. Li, G. Meng, L. Su, A. Chen, M. Xia, C. Xu, D. Yu, A. Jiang, J. Wei, Dichloroacetate blocks aerobic glycolytic adaptation to attenuated measles virus and promotes viral replication leading to enhanced oncolysis in glioblastoma, 2014. www.impactjournals.com/oncotarget/ (accessed March 24, 2020).

[60] J. Fernandez-de-Cossio-Diaz, A. Vazquez, Limits of aerobic metabolism in cancer cells, Sci. Rep. 7 (2017) 13488. https://doi.org/10.1038/s41598-017-14071-y.

[61] R.J. DeBerardinis, A. Mancuso, E. Daikhin, I. Nissim, M. Yudkoff, S. Wehrli, C.B. Thompson, Beyond aerobic glycolysis: Transformed cells can engage in glutamine metabolism that exceeds the requirement for protein and nucleotide synthesis, Proc. Natl. Acad. Sci. 104 (2007) 19345-19350. https://doi.org/10.1073/pnas.0709747104.

[62] R.J. DeBerardinis, T. Cheng, Q's next: the diverse functions of glutamine in metabolism, cell biology and cancer, Oncogene. 29 (2010) 313-324. https://doi.org/10.1038/onc.2009.358.

[63] C. Lee, F.M. Safdie, L. Raffaghello, M. Wei, F. Madia, E. Parrella, D. Hwang, P. Cohen, G. Bianchi, V.D. Longo, Reduced Levels of IGF-I Mediate Differential Protection of Normal and Cancer Cells in Response to Fasting and Improve Chemotherapeutic Index, Cancer Res. 70 (2010) 1564-1572. https://doi.org/10.1158/0008-5472.CAN-09-3228.

[64] C.H. O'Flanagan, L.A. Smith, S.B. McDonell, S.D. Hursting, When less may be more: calorie restriction and response to cancer therapy., BMC Med. 15 (2017) 106.

https://doi.org/10.1186/s12916-017-0873-x.

[65] D. Berrigan, J.A. Lavigne, S.N. Perkins, T.R. Nagy, J.C. Barrett, S.D. Hursting, Phenotypic effects of calorie restriction and insulin-like growth factor-1 treatment on body composition and bone mineral density of C57BL/6 mice: implications for cancer prevention., In Vivo. 19 (n.d.) 667-74. http://www.ncbi.nlm.nih.gov/pubmed/15999532 (accessed September 12, 2019).

[66] M.N. Harvie, A.H. Sims, M. Pegington, K. Spence, A. Mitchell, A.A. Vaughan, J.W. Allwood, Y. Xu, N.J.W. Rattray, R. Goodacre, D.G.R. Evans, E. Mitchell, D. McMullen, R.B. Clarke, A. Howell, Intermittent energy restriction induces changes in breast gene expression and systemic metabolism, Breast Cancer Res. 18 (2016) 57. https://doi.org/10.1186/s13058-0160714-4.

[67] M.N. Harvie, M. Pegington, M.P. Mattson, J. Frystyk, B. Dillon, G. Evans, J. Cuzick, S.A. Jebb, B. 
Martin, R.G. Cutler, T.G. Son, S. Maudsley, O.D. Carlson, J.M. Egan, A. Flyvbjerg, A. Howell, The effects of intermittent or continuous energy restriction on weight loss and metabolic disease risk markers: a randomized trial in young overweight women, Int. J. Obes. 35 (2011) 714-727. https://doi.org/10.1038/ijo.2010.171.

[68] J. Most, V. Tosti, L.M. Redman, L. Fontana, Calorie restriction in humans: An update, Ageing Res. Rev. 39 (2017) 36-45. https://doi.org/10.1016/j.arr.2016.08.005.

[69] S. de Groot, H. Pijl, J.J.M. van der Hoeven, J.R. Kroep, Effects of short-term fasting on cancer treatment., J. Exp. Clin. Cancer Res. 38 (2019) 209. https://doi.org/10.1186/s13046-0191189-9.

[70] L. Raffaghello, C. Lee, F.M. Safdie, M. Wei, F. Madia, G. Bianchi, V.D. Longo, Starvationdependent differential stress resistance protects normal but not cancer cells against highdose chemotherapy, Proc. Natl. Acad. Sci. 105 (2008) 8215-8220. https://doi.org/10.1073/pnas.0708100105.

[71] C. Lee, L. Raffaghello, S. Brandhorst, F.M. Safdie, G. Bianchi, A. Martin-Montalvo, V. Pistoia, M. Wei, S. Hwang, A. Merlino, L. Emionite, R. de Cabo, V.D. Longo, Fasting Cycles Retard Growth of Tumors and Sensitize a Range of Cancer Cell Types to Chemotherapy, Sci. Transl. Med. 4 (2012) 124ra27-124ra27. https://doi.org/10.1126/scitranslmed.3003293.

[72] S. Esaki, S.D. Rabkin, R.L. Martuza, H. Wakimoto, Transient fasting enhances replication of oncolytic herpes simplex virus in glioblastoma., Am. J. Cancer Res. 6 (2016) 300-11. http://www.ncbi.nlm.nih.gov/pubmed/27186404 (accessed September 12, 2019).

[73] G. Scheubeck, S. Berchtold, I. Smirnow, A. Schenk, J. Beil, U.M. Lauer, Starvation-Induced Differential Virotherapy Using an Oncolytic Measles Vaccine Virus, Viruses. 11 (2019) 614. https://doi.org/10.3390/v11070614.

[74] A.M. Al-Shammari, A.H. Abdullah, Z.M. Allami, N.Y. Yaseen, 2-Deoxyglucose and Newcastle Disease Virus Synergize to Kill Breast Cancer Cells by Inhibition of Glycolysis Pathway Through Glyceraldehyde3-Phosphate Downregulation, Front. Mol. Biosci. 6 (2019) 90. https://doi.org/10.3389/fmolb.2019.00090.

[75] G. Meng, B. Li, A. Chen, M. Zheng, T. Xu, H. Zhang, J. Dong, J. Wu, D. Yu, J. Wei, Targeting aerobic glycolysis by dichloroacetate improves Newcastle disease virus-mediated viroimmunotherapy in hepatocellular carcinoma, Br. J. Cancer. (2019). https://doi.org/10.1038/s41416-019-0639-7.

[76] R. V. Pusapati, A. Daemen, C. Wilson, W. Sandoval, M. Gao, B. Haley, A.R. Baudy, G. Hatzivassiliou, M. Evangelista, J. Settleman, MTORC1-Dependent Metabolic Reprogramming Underlies Escape from Glycolysis Addiction in Cancer Cells, Cancer Cell. 29 (2016) 548-562. https://doi.org/10.1016/j.ccell.2016.02.018.

[77] L.D. Marroquin, J. Hynes, J.A. Dykens, J.D. Jamieson, Y. Will, Circumventing the Crabtree Effect: Replacing Media Glucose with Galactose Increases Susceptibility of HepG2 Cells to Mitochondrial Toxicants, (n.d.). https://doi.org/10.1093/toxsci/kfm052.

[78] V.M. Gohil, S.A. Sheth, R. Nilsson, A.P. Wojtovich, J.H. Lee, F. Perocchi, W. Chen, C.B. Clish, C. Ayata, P.S. Brookes, V.K. Mootha, N.B. Author, Discovery and therapeutic potential of drugs that shift energy metabolism from mitochondrial respiration to glycolysis HHS Public Access Author manuscript, Nat Biotechnol. 28 (2010) 249-255. https://doi.org/10.1038/nbt.1606.

[79] M. Potter, E. Newport, K.J. Morten, The Warburg effect: 80 years on, (2016). https://doi.org/10.1042/BST20160094. 
[80] D.B. Rivadeneira, K. DePeaux, Y. Wang, A. Kulkarni, T. Tabib, A. V. Menk, P. Sampath, R. Lafyatis, R.L. Ferris, S.N. Sarkar, S.H. Thorne, G.M. Delgoffe, Oncolytic Viruses Engineered to Enforce Leptin Expression Reprogram Tumor-Infiltrating T Cell Metabolism and Promote Tumor Clearance, Immunity. 51 (2019) 548-560.e4.

https://doi.org/10.1016/j.immuni.2019.07.003. 